\title{
Corpo estranho em trato digestivo em crianças: conduta na emergência
}

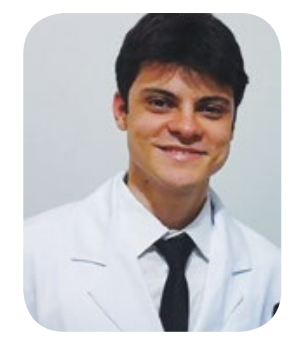

Ian Freitas Simões ${ }^{1}$, Dilton Rodrigues Mendonça ${ }^{2}$, Marcos Clarêncio Batista Silva ${ }^{2}$, Soraya Fernanda Cerqueira Motta $^{2}$, Rita Mira de Oliveira ${ }^{3}$

\section{INTRODUÇÃO}

A ingestão de corpo estranho é uma ocorrência comum em crianças. Geralmente, ocorre passagem e eliminação espontânea do objeto através do trato gastrointestinal e apenas uma pequena parcela dos casos necessitam de intervenção endoscópica ou cirúrgica. Apesar do grande número de casos, a morbidade grave e a mortalidade são muito pequenas e geralmente estão associadas à obstrução ou perfuração do trato digestivo. Estima-se que, nos Estados Unidos, anualmente mais de 100.000 crianças são atendidas no serviço de emergência após ingestão de corpo estranho ${ }^{1,5}$.

A maioria dos casos (mais de 90\%) ocorre em crianças entre seis meses e seis anos de idade ${ }^{1,2,3,5}$. A ingestão geralmente é acidental e os objetos ingeridos normalmente são encontrados no ambiente doméstico, como brinquedos, moedas, bijuterias, pilhas e alimentos. Em alguns pacientes psiquiátricos, pode haver a ingestão provocada ou intencional.

Alguns objetos merecem atenção especial devido à sua toxicidade e risco potencial de lesão aguda do trato digestivo, morbidade grave e mortalidade. Entre as lesões mais preocupantes e potencialmente catastróficas estão as perfurações e fístulas digestivas, sendo mais frequentes após ingestão de pilhas, objetos perfurantes e múltiplos ímãs.

Quando a impactação ocorre no esôfago, ela geralmente se dá ao nível dos estreitamentos fisiológicos, como esfíncter esofágico superior (músculo cricofaríngeo), impressão do arco aórtico e esfíncter esofágico inferior ${ }^{1,4,5,9}$. Objetos alojados na porção média do esôfago têm maior probabilidade de induzir espasmos e causar lesões. Tais lesões são temidas, pois podem levar a graves complicações como as estenoses, perfurações e fístulas para o aparelho respiratório. Objetos perfurantes podem lesar o esôfago, levando a edema cervical, crepitações do subcutâneo ou pneumomediastino. Lesões próximas à aorta podem levar a sangramentos fatais.

Ao passarem pelo esôfago, 80 a $90 \%$ dos objetos ingeridos são eliminados espontaneamente, sem necessidade de conduta adicional. As manifestações dessas impactações são geralmente tardias ${ }^{1,5,8,10}$. Entretanto, alguns pacientes precisarão de intervenção, a depender da sua idade, status clínico e tipo de objeto ingerido. Neste cenário, a endoscopia digestiva desempenha papel fundamental, pois é capaz de minimizar os índices de lesões digestivas agudas e suas sequelas, como as estenoses de esôfago. Em menos de $20 \%$ dos casos haverá indicação formal de remoção endoscópica, sendo necessária especialmente quando há impactação em algum local do trato digestivo ou potencial complicação pela presença do objeto.

O médico que atende a criança na emergência com suspeita ou confirmação de ingestão de corpo estranho deve estar atento para a necessidade e o momento de indicar a realização da endoscopia digestiva, podendo indicá-la em regime de urgência, emergência ou ambulatorial.

Quando se opta pela conduta expectante, os seguintes marcos anatômicos devem ser cuidadosamente avaliados: esfíncter esofágico superior, esfíncter esofágico inferior, piloro, curvatura do duodeno e ângulo de Treitz (Fig.1). Nesses locais, a impactação é mais frequente. Os pacientes portadores de patologias ou cirurgiasgastrointestinais prévias têm maior risco de impactação, perfuração e obstrução. 


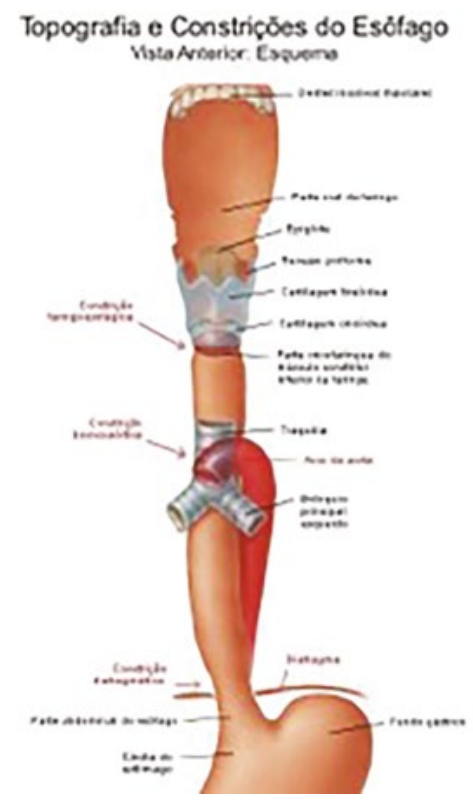

Figura 1 - Anatomia do esôfago - Atlas de Anatomia Frank Netter, $5^{\mathrm{a}}$ edição

\section{DIAGNÓSTICO}

\section{História clínica e exame físico}

$\mathrm{Na}$ maioria dos casos existe o relato ou o testemunho da ingestão do corpo estranho. Em crianças menores ou portadoras de distúrbios cognitivos, no entanto, o diagnóstico pode ser mais difícil e necessita de uma abordagem mais detalhada da história clínica.Os achados clínicos dependem da idade da criança, natureza do objeto e da região anatômica envolvida. Por isso, deve-se sempre manter alto grau de suspeita nas crianças que se apresentam muito sintomáticas e de forma brusca com tosse, engasgo, sialorreia, vômitos, dificuldade respiratória, sangramento digestivo, dor torácica e recusa alimentar.O objeto ingerido deve ser avaliado quanto ao tipo, forma, tamanho, toxicidade e localização no trato digestivo.

O exame físico deve estar voltado para a detecção de complicações. Pacientes com história compatível e hemodinamicamente instáveis devem ser prontamente avaliados pela equipe cirúrgica. Via aérea e respiração são prioridades. Edema, eritema e crepitação na região cervical indicam perfuração esofágica. Estridor e sibilância podem indicar impactação digestiva, causando compressão extrínseca do aparelho respiratório ${ }^{5,7,10}$. Pacientes com distensão abdominal, dor intensa ou sinais de abdome agudo perfurativo ou obstrutivo devem ser submetidos a exames de imagem (radiografia) e avaliados pela equipe de cirurgia.

\section{EXAMES COMPLEMENTARES Radiografia simples}

A radiografia simples de tórax ântero-posterior e lateral tem papel fundamental na avaliação de objetos radiopacos, especialmente na diferenciação entre pilhas e moedas. Este exame deve ser realizado durante a avaliação inicial ou investigação de todos os casos de ingestão de corpo estranho, mesmo naqueles considerados radiotransparentes, pois a condução de cada caso dependerá da sua localização, formato e tamanho do objeto ${ }^{5,7}$. Nos objetos não radiopacos, a radiografia simples pode fornecer sinais indiretos de perfuração ou identificar outras complicações.

Quando há dúvidas ou incertezas diagnósticas, pode-se utilizar radiografias de abdome e região cervical superior para complementar a investigação. Para a diferenciação radiológica entre pilhas e moedas deve-se procurar pela imagem do duplo contorno (duplo anel ou duplo halo) na imagem ântero-posterior o pelo sinal "step off" na imagem em perfil. Objetos planos geralmente apresentam orientação no plano coronal, aparecendo como objetos circulares na projeção ântero-posterior (Fig.2 e Fig.3).

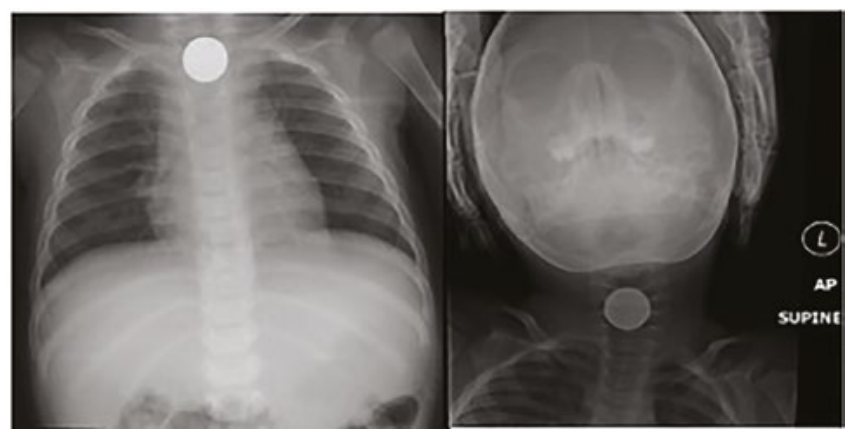

Figuras 2 e 3 - Imagem de moeda impactada no esôfago superior. Bateria localizada no esôfago superior. http:// emedicine.medscape.com/article/408752-overview.

Além da sua utilidade para identificação do corpo estranho ingerido, a radiografia simples de tórax e abdome pode fornecer indícios de obstrução e perfuração do trato digestivo, como: pneumoperitônio, pneumomediastino, distribuição inadequada de gases ou distensão de alças com nível líquido. Outra aplicação do método ocorre no seguimento periódico para os pacientes que serão acompanhados ambulatorialmente. Nestes casos, radiografias de tórax/abdome podem ser realizadas a cada 3 a 5 dias, no intuito de constatar a progressão do objeto através do trato digestivo. 
Objetos que permanecem no mesmo local anatômico, por um período superior a 3 dias, pode-se considerar a remoção'.

O exame contrastado raramente será utilizado, tendo indicação na investigação posterior de perfurações do trato digestivo. Nesses casos utiliza-se contrastes hidrossolúveis, contendo iodo, uma vez que os contrastes feitos com bário estão formalmente contraindicados. Além disso, o bário pode prejudicar a visualização endoscópica posterior.

\section{Tomografia computadorizada}

A tomografia raramente será indicada para avaliação inicial. Pode ser indicada na suspeita de complicações, como perfurações, obstruções, fístulas e complicações em estruturas adjacentes. Existe indicação quando suspeita de complicações após remoção do corpo estranho. Não deve ser realizado rotineiramente na avaliação inicial.

Quando, durante a avaliação inicial, o objeto não é visto à radiografia de tórax (ex. radiotransparente), mas o paciente encontra-se sintomático, o objeto possui características preocupantes (longo, largo ou perfurante) ou suas características não são conhecidas, este exame pode ser realizado ${ }^{5}$.

\section{Ressonância nuclear magnética}

A ressonância não tem indicação na avaliação inicial. Pode ser utilizada, em raras situações, na pesquisa de complicações secundárias à ingestão. Quando o objeto é radiolucente e não é visto à radiografia de tórax, a ressonância pode ser utilizada em substituição à tomografia. Este exame é formalmente contraindicado na investigação de objetos metálicos ${ }^{5}$.

\section{Endoscopia digestiva}

A endoscopia pode ser um método tanto diagnóstico quanto terapêutico. É o método de escolha para a remoção de objetos, pois permite visualização direta do corpo estranho e avaliação simultânea do trato digestivo circunjacente. Sua realização pode ocorrer em regime de emergência (máximo de duas horas da ocorrência) ou urgência (até $24 \mathrm{~h}$ do acidente) ${ }^{1}$. A indicação deve ser criteriosa, pois o exame realizado em regime de emergência não permite o jejum adequado, aumentando o risco de broncoaspiração. As principais indicações de endoscopia de emergência são ${ }^{1,2,3,5,11}$ :

- bateria localizada no esôfago;

- ingestão de múltiplos ímãs ou ímãs associados a peças metálicas;

- objeto impactado no esôfago (maior risco em crian- ças menores de 5 anos e objetos maiores que $2 \mathrm{~cm}$ );

- risco de aspiração (com o paciente incapaz de deglutir saliva);

- objetos perfurantes ou cortantes;

- pacientes muito sintomáticos (especialmente se há sintomas respiratórios).

Principais indicações de remoção em regime de urgência ${ }^{1,2,3,5,11}$ :

- objetos localizados no estômago maiores que 2,5 $\mathrm{cm}$ de diâmetro (maior chance de impactar no piloro) ou maiores que $6 \mathrm{~cm}$ de comprimento;

- não progressão do objeto localizado no estômago por mais de $24 \mathrm{~h}$;

- persistência dos sintomas esofágicos, mesmo se o objeto estiver localizado no estômago.

Quando se opta pela indicação de endoscopia em regime de urgência para remoção de objetos localizados no esôfago, é prudente a realização de uma nova radiografia imediatamente antes do procedimento. Tal prática se justifica pela observação que grande parte dos objetos impactados no esôfago migrarem para o estômago durante o período de espera ou preparo para o procedimento. Desta forma, deixa-se de realizar endoscopias desnecessárias.

Alguns pacientes podem ser acompanhados ambulatoriamente, sem necessidade de intervenção endoscópica. Deve-se assegurar o seguimento e reavaliações periódicas do paciente. Seu acesso ao serviço de saúde deve ser simples e rápido. Nos casos em que não se pode assegurar o seguimento ambulatorial, deve-se considerar o internamento. Não existe indicação de medicamentos laxantes ou que aumentem o trânsito intestinal. Deve-se aumentar a ingestão de líquidos e alimentos ricos em fibras. Os cuidadores devem estar atentos à eliminação do objeto via fecal.

Qualquer paciente que apresente sintomas de sialorreia, dor abdominal, sangramento digestivo, náuseas e vômitos persistentes deve ser prontamente reavaliado e considerada a realização de estudos por imagem e/ou endoscopia.

Objetos de baixo risco não precisam ser acompanhados com radiografias seriadas ${ }^{1,3,5}$. Em alguns casos, o exame radiográfico pode ser repetido a cada 3-5 dias, podendo-se realizar a remoção cirúrgica ou endoscópica em casos de não progressão do objeto ${ }^{1,3,5}$.

\section{CIRURGIA}

Poucos pacientes necessitarão de intervenção cirúrgica. Nos pacientes muito sintomáticos, especialmente naqueles com instabilidade hemodinâmica, o 
seguimento inicial deve ser realizado em parceria com a equipe de cirurgia. Quando se detecta perfuração esofágica durante a avaliação endoscópica, procede-se com internação, passagem de sonda nasoenteral, dieta zero e antibioticoterapia profilática ${ }^{1,2,3}$. Nesses casos, a avaliação cirúrgica deve ser precoce, a fim de evitar e minimizar sequelas. Os casos de complicações como estenoses, perfurações, obstruções e fístulas devem ser avaliados individualmente e a intervenção cirúrgica deve ser sempre considerada.

\section{FLUXOGRAMAS}
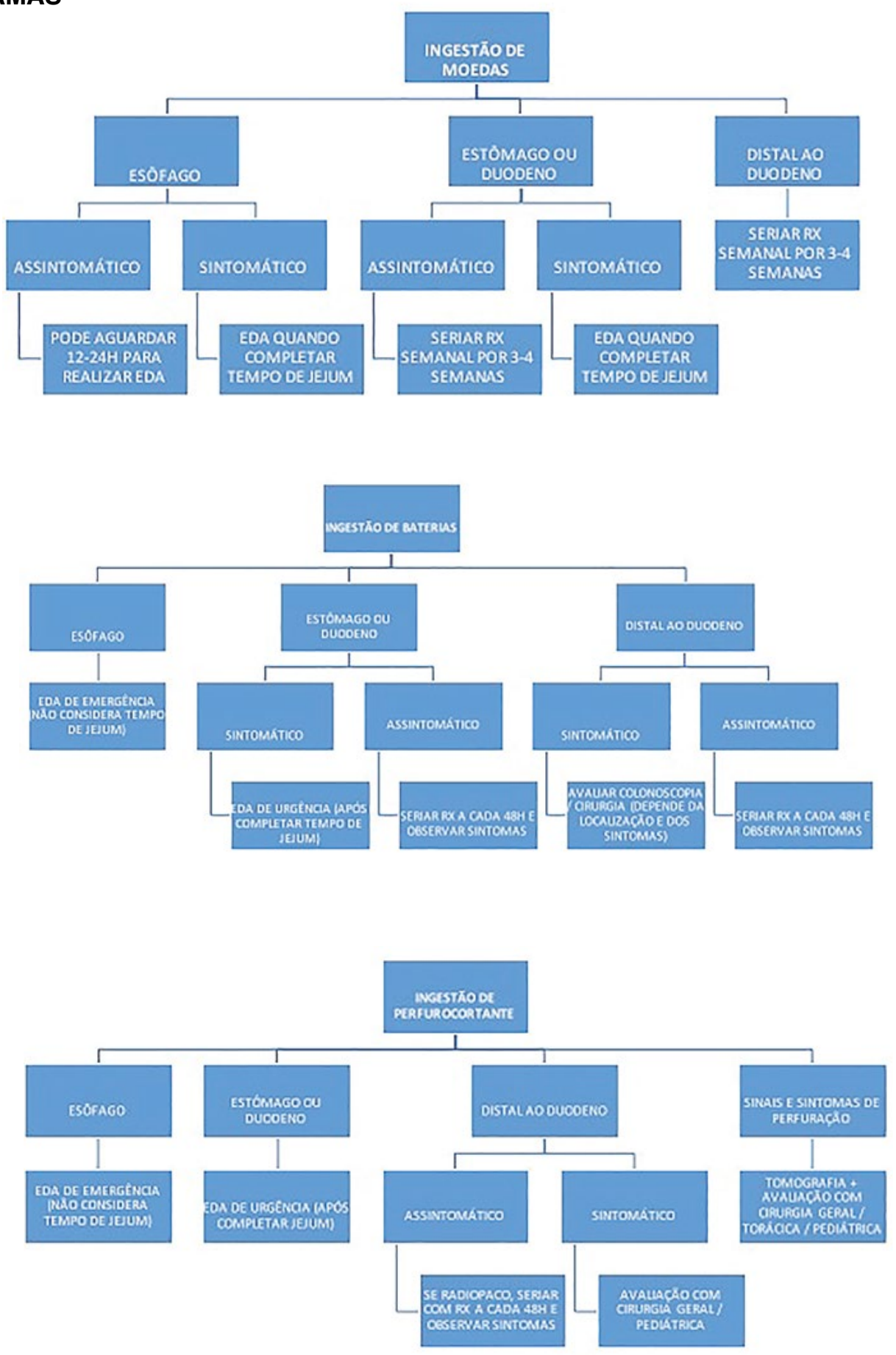


\section{REFERÊNCIAS}

1. Management of Ingested Foreign Bodies in Children: A Clinical Report of the NASPGHAN Endoscopic Committee. JPGN Volume 60, Number 4, April 2015

2. Emerging Battery-Ingestion Hazard: Clinical Implications; PEDIATRICS. Volume 125, Number 6, June 2010.

3. Preventing Battery Ingestion: An Analysis of 8648 Cases; PEDIATRICS. Volume 125, Number 6, June 2010.

4. Management of ingested foreign bodies and food impactions. GASTROINTESTINAL ENDOSCOPY. Volume 73, No. $6: 2011$

5. Mark A Gilger, MD; Ajay K Jain, MD; Mark E Mc Omber, MD. Foreign bodies of the esophagus and gastrointestinal tract in children. UpTodate. Disponível em http://www.uptodate.com/contents/foreign-bodies-of-the- esophagus-and-gastrointestinal-tract-inchildren.

6. KIRKHAM, EN; KANE, M; PAUL, SP. Foreignbodyingestion in children. Community practitioner: the journal of the community practitioners' \& health visitors' association. England, 88, 3, 45-48, Mar. 2015. ISSN: 1462-2815.

7. SINK, JR; et al. Diagnosis of pediatric foreign body ingestion: clinical presentation, physical examination, and radiologic findings. The Annals Of Otology, Rhinology, And Laryngology. United States, 125, 4, 342-350, Apr. 2016. ISSN: 0003-4894.

8. JOYAMAHA, D; CONNERS, GP. Managing pediatric foreign body ingestions. Missouri Medicine. United States, 112, 3, 181-186, May 2015. ISSN: 00266620.

9. ANTONIOU, D; CHRISTOPOULOS-GEROULANOS, G. Management of foreign body ingestion and food bolusimpaction in children: a retrospective analysis of 675 cases. The Turkish Journal Of Pediatrics. Turkey, 53, 4, 381-387, July 2011. ISSN: 0041-4301.

10. SINK, JR; et al. Diagnosis of pediatric foreign body ingestion: clinical presentation, physical examination, and radiologic findings. The Annals Of Otology, Rhinology, And Laryngology. United States, 125, 4, 342-350, Apr. 2016. ISSN: 0003-4894.

11. LEE, JH; et al. Foreign body ingestion in children: should button batteries in the stomach be urgently removed? Pediatric Gastroenterology, Hepatology \& Nutrition. Korea (South), 19, 1, 20-28, Mar. 2016. ISSN: 2234-8646.
1- Programa de Residência Médica de Pediatria $\mathrm{HCRS} / \mathrm{HSI}$

2- Serviço de Pediatria do Hospital Central Roberto Santos

3- Serviço de Pediatria do HSI

Endereço para correspondência:

ian_simoes@hotmail.com 